\title{
A cloud-based approach for WEEE remanufacturing
}

\author{
Lihui Wang (2) $)^{\mathrm{a},}$, Xi Vincent Wanga, Liang Gaob, József Váncza (1)c,d \\ a Department of Production Engineering, KTH Royal Institute of Technology, Sweden \\ ${ }^{\mathrm{b}}$ School of Mechanical Science and Engineering, Huazhong University of Science and Technology, China \\ ${ }^{c}$ Institute for Computer Science and Control, Hungarian Academy of Sciences, Hungary \\ d Dept. of Manufacturing Science and Technology, Budapest University of Technology and Economics, Budapest, Hungary
}

Abstract: The modern manufacturing industry calls for a new generation of integration models that are more interoperable, intelligent, adaptable and distributed. Evolved from service-oriented architecture, web-based manufacturing and cloud computing, cloud manufacturing is considered worldwide a new enabling technology for manufacturing enterprises to respond quickly and effectively to the changing global market. For Waste Electrical and Electronic Equipment (WEEE) in particular, it is a critical necessity to recycle, reuse and remanufacture WEEE products by setting up a cloud-based information system. In this paper, a novel service-oriented remanufacturing platform is proposed based on the cloud manufacturing concept.

Keywords: Recycling, Service, Cloud manufacturing

\section{Introduction}

Due to the rapid changes in technologies, shifting in media (tape, CD, DVD, MP3, etc.) and falling prices, the total volume of electronic wastes is growing fast globally. On the other hand, planned obsolescence that shortens the lifecycle of the electronic devices brings huge profits to the vendors. According to the statistics of the environmental protection agency of the USA, new electronic products sold in 2009 doubled the number since 1997 [1]. Unfortunately, only $25 \%$ of end-of-life (EOL) products were collected for recycling. Waste Electrical and Electronic Equipment (WEEE) belongs to this type of discarded devices. The processes of dismantling and disposing of electronic wastes lead to huge environmental impacts globally. In the EU, it is a highpriority mission to collect, recycle and recover all types of WEEE devices in all member states.

\section{Background}

In recent years, international directives and national standards have been published to define the treatment routes after an Electrical and Electronic Equipment (EEE) reaches the end phase of its lifecycle and turns into WEEE. In general, the status of a WEEE is individually evaluated on the product, component, and material levels. Based on the evaluation results, they can be further directed to different routes [2-4]. A generalised WEEE roadmap for this purpose is presented in Fig. 1.

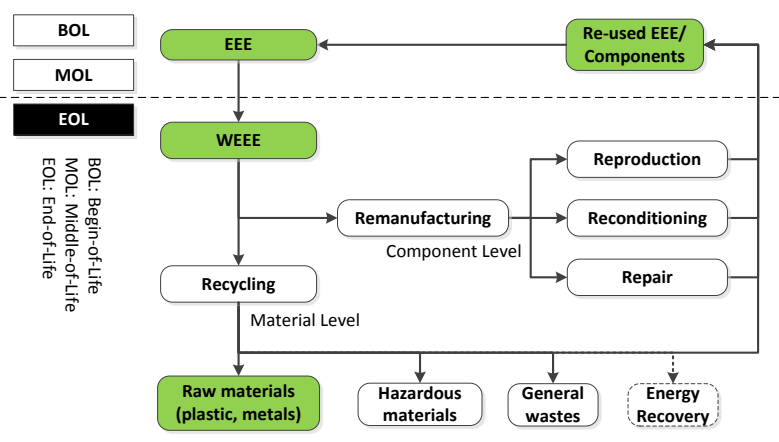

Fig. 1. Generalised WEEE recovery flow.

The treatment routes belong to two broad categories: recycling at the material level and remanufacturing at the component level. The recycling process aims at collecting the materials left in WEEE, including plastic, metal, etc., while remanufacturing, as discussed in this paper, restores the functionality of products or their components. It includes reproduction, recondition and repairing. Based on the different evaluation results of the individual WEEE, the product or its components will be reused, reconditioned or repaired before they are issued with new warranty conditions.

The profitable business opportunities can be summarised as a smiling curve [5], showing a high profitability on the downstream phase of products (see Fig. 2). However, tail management of the WEEE lifecycle is difficult in particular because the information control of the products is normally interrupted when the product flow comes to end users. The end users are scattered both in time and space. The owners of ordinary EEE such as TV sets, computers, smartphones, etc., normally do not possess sufficient knowledge about remanufacturing. When these products reach the EOL phase, it is hard to feedback their status to the original equipment manufacturers (OEMs) or remanufacturers. Targeting the problem, in this research, a novel remanufacturing system is developed to support WEEE remanufacturing. With the help of an integrated cloud-based infrastructure, it is especially dedicated to support bidirectional communication at the EOL stage.

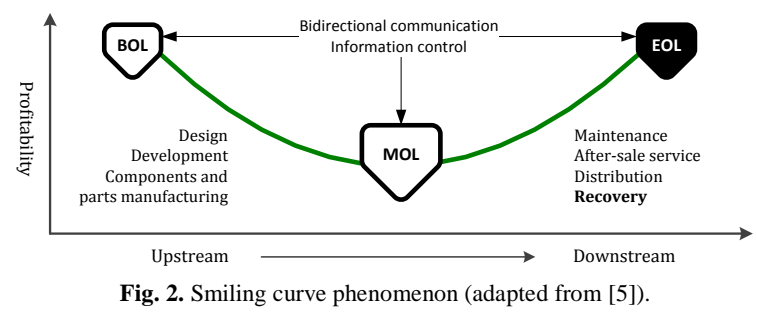

\section{From cloud manufacturing to cloud remanufacturing}

Recently, cloud computing has changed the way of thinking of both ICT providers and their customers. It offers business and application models that deliver infrastructure, platform, software and applications in forms of services [6]. Inspired by the success of cloud computing, the cloud concept has been extended to the manufacturing contexts, leading to the innovation of various cloud manufacturing systems $[7,8]$. Cloud manufacturing implies an integrated cyber-physical system that can provide on-demand manufacturing services, digitally and physically, at the best utilisation of manufacturing resources. It aims at offering a shared pool of resources such as manufacturing software tools, manufacturing facilities, and manufacturing capabilities. However, cloud manufacturing is more than simply deploying manufacturing software applications in the computing cloud. Besides data storage and virtual machines, the physical resources 
integrated in the manufacturing cloud are able to offer adaptive, secure and on-demand manufacturing services over the Internet of Things, including workcells, machine tools, robots, etc.

Although product recovering offers new links between different types of businesses and industries [9], interrupted information exchange at EOL phase is still the main hindrance to developing an integrated and collaborative remanufacturing environment as mentioned above. To overcome this bottleneck, it is possible to introduce the cloud concept into the remanufacturing context. A service-oriented environment like cloud can integrate the current remanufacturing capabilities and most importantly, facilitate the provision of a comprehensive and standardised data exchange environment that maintains all the information and knowledge throughout the EEE lifecycle.

The data exchange and management framework is illustrated in Fig. 3. For WEEE, data and knowledge maintenance starts from EEE product registration at the Begin-of-Life (BOL) phase, when its OEM is responsible for establishing and maintaining the product details and component specifications. The information is integrated with the product data model in the Cloud Storage. Additionally, bill of materials is also maintained, which is especially valuable for later remanufacturing or recycling operations. In addition, special treatments are organised based on the hazardous element list of the WEEE product, and disassembly services are assigned specifically for the valuable components and materials. After an EEE reaches the Middle-ofLife (MOL) stage, the retailers and customers play important roles in registering sold products and updating their EEE status.

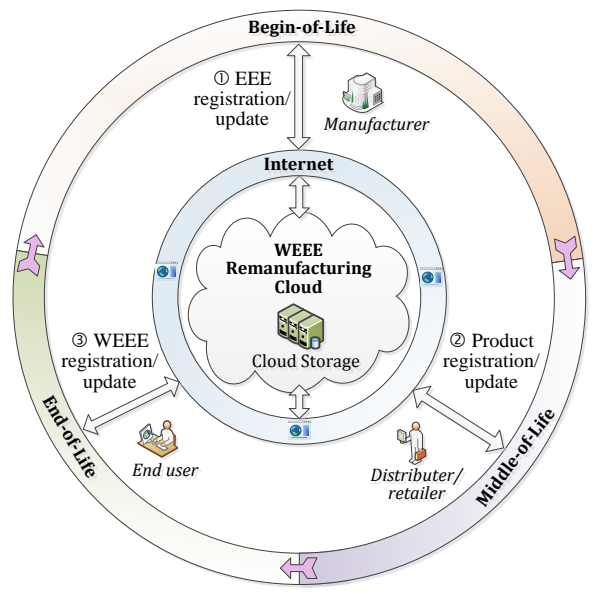

Fig. 3. Cloud-based WEEE data management.

At the EOL phase, the end users are neither expected nor required to possess any expertise in recycling or remanufacturing. They can interact with the cloud remanufacturing environment via web browsers or mobile apps to update the latest status of products, including their registration as WEEE once they stop service. The WEEE registration is then forwarded to the remanufacturing cloud. Consequently, related remanufacturing services are organised accordingly. In this way, comprehensive product knowledge and history is integrated in the Cloud Storage from-cradle-to-cradle, which overcomes the communication bottlenecks over different lifecycle phases and among multiple stakeholders.

\section{Cloud-based WEEE remanufacturing system}

This section discusses the architecture of a novel WEEE Remanufacturing Cloud (WRCloud), as depicted also in Fig. 4. In this three-layer architecture, the remanufacturing resources and their capabilities are provisioned in terms of cloud remanufacturing services. The end users are connected to the WRCloud system at the User Layer. Note that in the WRCloud, users are defined as the stakeholders who demand cloud services. They can be either EEE customers at MOL phase or WEEE remanufacturing participants at EOL phase, looking for collaboration. The browser-based user interfaces collect varying

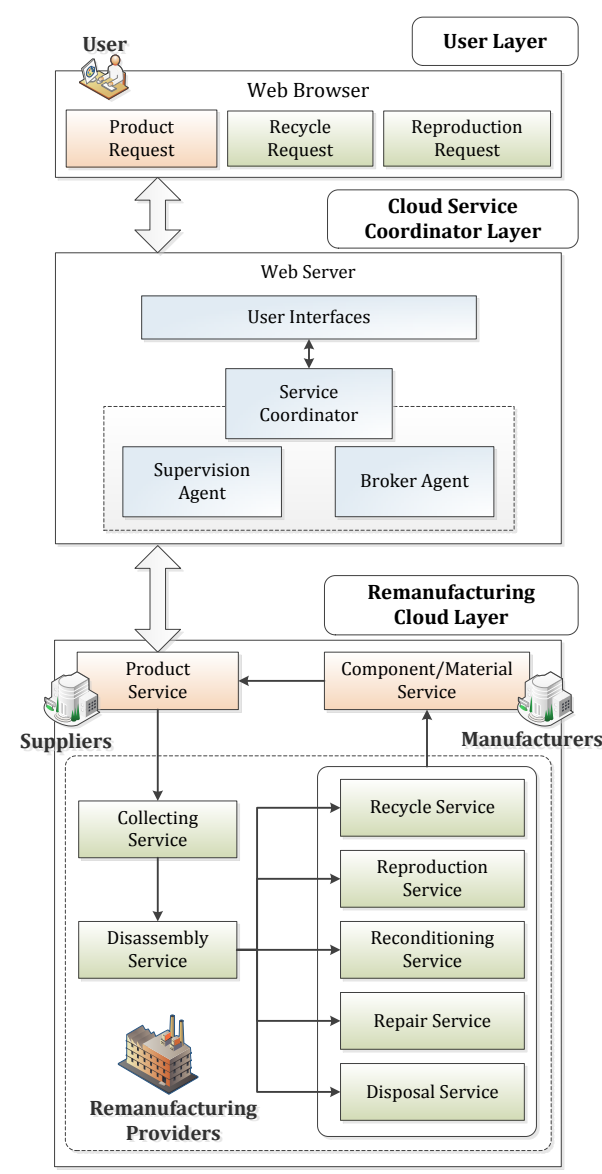

Fig. 4. Three-layer WRCloud.

service requests and document them in a standardised format. The documented service requests are then delivered to the Cloud Service Coordinator Layer.

The Service Coordinator plays the role of an orchestrator of the entire cloud. It is developed by making use of software agent technology. The Broker Agent is responsible for interpreting service requests from the end users and looking for appropriate service solutions accordingly. The available service packages are broadcasted in the cloud database with explicit technical specifications such as cost, time, resource consumptions, etc. Departing from these specifications, feasible service suggestions are delivered to the user for review, negotiation and confirmation. Eventually, a service roadmap is delivered to the Supervision Agent who executes it step-by-step. From the users' point of view, their original service demands are fulfilled by the WRCloud as a whole. The users interact with the Service Coordinator via interfaces without direct involvements in the operation of remanufacturing resources at the lower layer. In fact, they do not need to be informed about the details of the remanufacturing activities. The users are supported by an everything-as-a-service (XaaS) model that is realised by the service providers in the cloud. Therefore, the WRCloud provides a service-oriented "request-find-provide" business model. In the cloud environment, the Cloud Coordinator helps synthesise and integrate up-to-date information from various sources, and presents them in a well-structured way to the users. It is the cloud users who make final decisions on whether to go for reproduction, reconditioning or repair, and if so, with which service providers.

In order to establish comprehensive service management, various mechanisms are deployed at the Service Coordinator Layer, like WEEE tracking, evaluation, and standardised description mechanism.

\subsection{QR code based WEEE tracking mechanism}

The end users of EEE are ordinary civilians who are normally lacking expertise of and resources for remanufacturing or 
recycling. After their EEE devices reach the end stage of their lifecycle, in most cases it is not practical for the end users to identify the products' specifications and organise remanufacturing service individually. This leads to the aforementioned communication bottleneck. To further improve the performance and portability of WRCloud, the data integration can be supported by the Quick Response Code (QR Code). A QR code is a machine-readable label, which is represented by a matrix barcode. In the recent years, the data storage capacity of $\mathrm{QR}$ codes has been greatly improved. These labels can be attached to EEE products or even to the critical components inside. When the products reach the EOL phase, the users can scan the QR code labels on the products using smartphones, cameras, or PDAs (Fig. 5). The WRCloud is then able to interpret these QR codes and identify the WEEE specifications accordingly. Based on the data stored in a QR code, detailed WEEE and its related BOL/MOL history in the Cloud Storage can be quickly located and connected to the specifications of products in the users' hands. Thus the cloud provides a complete history and background of the products with full technical details. With the support of such information, a customised remanufacturing service is then organised based on the personalised settings and contents from the users. This way, the broken information control between end users and high-level decision makers can be rebuilt, thanks to the QR-based product tracking approach.

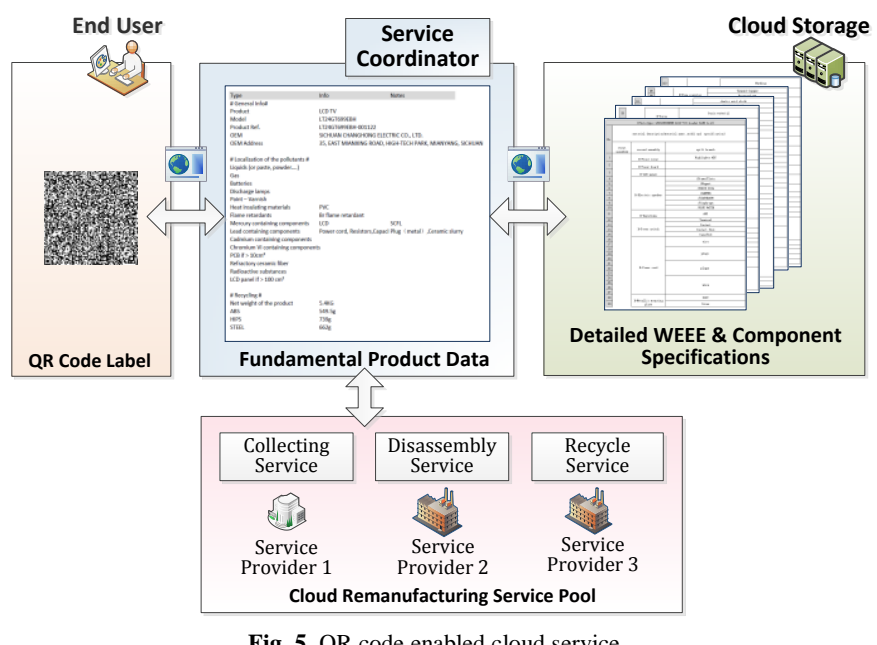

4.2 Service evaluating mechanism based on lifecycle assessment

For decision making in WRCloud, the Service Coordinator needs to assess both economic and environmental impacts in order to achieve sustainable service solutions. Narrow outlook on service scheduling should be avoided, by associating it with all stages of a product's lifecycle. Thus in this research, lifecycle assessment (LCA) mechanism is deployed in the Service Coordinator Layer. Here, LCA aims at comparing likely environmental impacts, climate change effects and human health effects due to different cloud services in order to provide a sound basis for informed decisions on a cloud service roadmap. According to the LCA assessment, service plans can be identified and reviewed in a quantifiable manner, including environmental impacts and human health damages. Furthermore, multiple cloud service roadmaps can be delivered to and compared by customers and service providers alike. Hence, the Service Coordinator can develop the most sustainable service solutions based on LCA results. More details on the QR tracking and LCA analysis are presented in the case study in Section 5.

\subsection{Standardised cloud service description method}

In WRCloud, the product information is tracked by QR codes and service packages are evaluated by LCA. Furthermore, it is crucial to integrate and maintain the information and results in a standardised data environment. In the WRCloud, uniformed data models are developed and used to describe the important entities throughout the recovery and recycling chain, such as products, WEEE, end users, service providers, cloud services, and so forth (Fig. 6). Standardised application protocol interfaces are developed to connect these models and provide an interoperable solution for the processes and interactions in the WRCloud. The core of the data models focuses on the cloud service data model, which documents what kind of remanufacturing/recycling service is requested and how it is achieved. The EEE data model is established on the basis of the current ISO 10303 standard series and the extensive developments on cloud manufacturing. The full product lifecycle is defined as a project model. Its BOL, MOL data from OEMs and retailers, as well as the EOL data from the QR tracking mechanism are all stored as the standardised data models in the Cloud Storage. Thus, it provides a uniformed data exchange environment for the WRCloud.

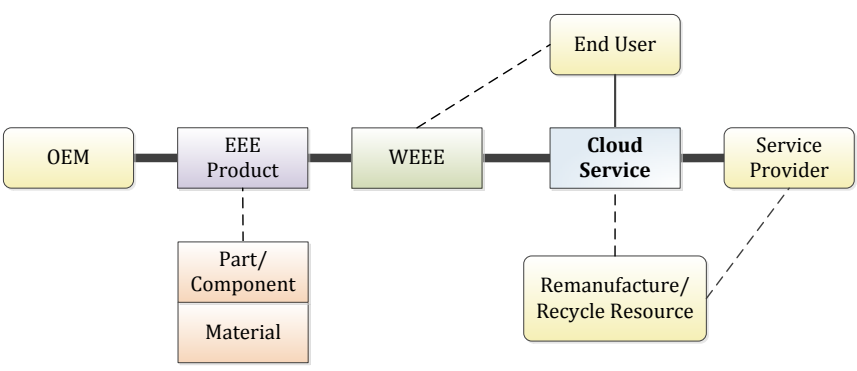

Fig. 6. Standardised data model set for cloud services

At the Remanufacturing Cloud Layer shown in Fig. 4, current remanufacturing resources and their capabilities are integrated as modularised service units. Based on the service roadmap from the Service Coordinator, the service units are strung accordingly as a combination of virtual services that can physically achieve the service objectives. When joining the remanufacturing cloud, the providers deliver services based on the agreements on quality of services, operation security, trust of collaborations, etc. [10]. WRCloud offers channels for information sharing and mechanisms for collaboration and process integration on high level. At this layer, multiplication of similar service packages is necessary, due to optimised solutions and availability.

Note that the roles of participants in cloud-based remanufacturing are different from those in conventional systems. In traditional web-based recovery or remanufacturing approaches, the users of a system/platform are mainly remanufacturers. The system helps them to communicate with remote resources via networks. In the cloud-based approach, on the other hand, the users of the system are the end users of EEEs. Remanufacturing stakeholders related to remanufacturing/ recycling processes play as the service providers in the WRCloud with responsibilities of collecting and reproducing products. Thus a many-to-many remanufacturing model can be established to integrate the stakeholders who are already involved in the remanufacturing and recycling business, instead of the traditional one-to-many approach.

The cloud-service providers are integrated as a shared pool of remanufacturing and recycling resources and capabilities. Thus the business risk is also shared in the whole environment. Despite the uncertainty and unpredictability of individual service demand, the aggregated demands can be planned and optimised in a remanufacturing environment like WRCloud that operates with look-ahead.

\section{Case study}

As a working example, a LCD TV (Fig. 7) is chosen as the WEEE in order to show the basic mechanisms of the proposed system. The basic information of the product is recorded in the QR code tag attached on the product as mentioned in Section 4.1. The owner is able to scan the code via a smartphone and submit the code together with service queries to the WRCloud. The user then links the latest WEEE status to the product profiles, and registers it as a WEEE. Based on the interpreted QR code, detailed product 
specifications can be quickly retrieved from the Cloud Storage. Related remanufacturing and recycling services are organised based on the information on both the material and component levels. Next, the Cloud Coordinator suggests the feasible service options to the user. Finally, the user requests a service package including collecting, disassembly and recycling services.

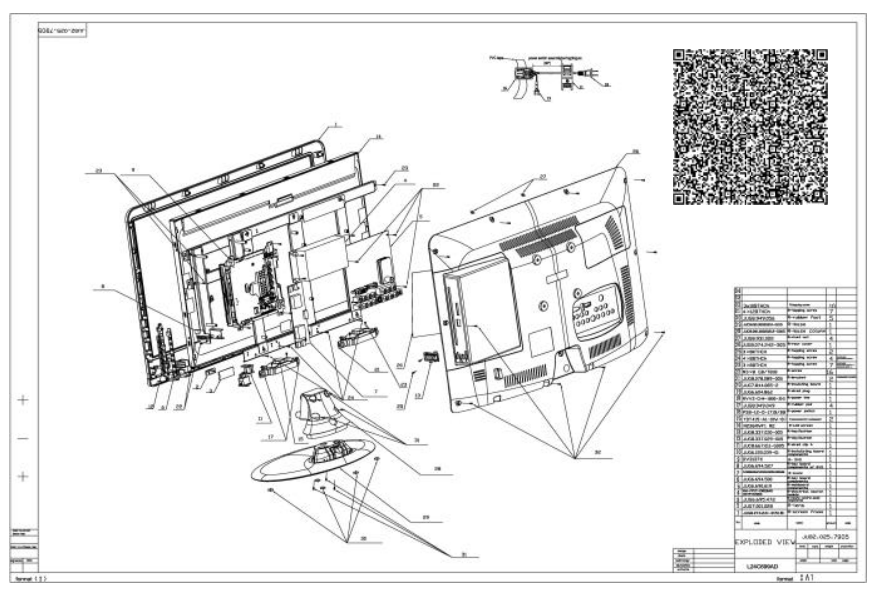

Fig. 7. Exploded view of an LCD TV.

During service negotiation, multiple disassembly options are available in the cloud. Based on the technical specifications, a 20operation service is scheduled to separate the components and materials of the LCD. Besides logistic evaluation, the economic and environmental impacts of the operations are obtained by the LCA mechanism inside the Service Coordinator. In this case study, one of the most widely used impact assessment methods, the socalled Eco-indicator 99 [11] is adopted. It allows for expressing the environmental load of a product in a single score. Accordingly, a simplified physical flow can be expressed as

$$
f=\frac{m}{t}=\lambda \cdot a \cdot p e c
$$

where $f$ is the simplified physical flow $[\mathrm{kg} /$ year], $\mathrm{m}$ the mass $[\mathrm{kg}]$, $t$ time [year], $\lambda$ a model constant [m/year], $a$ area $\left[\mathrm{m}^{2}\right]$, and pec predicted environmental concentration $\left[\mathrm{kg} / \mathrm{m}^{3}\right]$.

As shown in Fig. 8, the accumulated impact factors of these 20 disassembly operations are summarised together with operation time and directly recovered value. Avoided environmental impact indicates the points (Pt) that benefit the global environment from directly recovered materials (DRM), and avoided climate change effect is measured by DALY (Disability Adjusted Life Years). Due to the variations of currency and level of resources, the costs to achieve the same remanufacturing service are different pertinent to different service providers. In this case study, the accumulated labour cost in China is compared with that in UK. In the end, the Chinese service provider is chosen by the coordinator since it offers similar level of services with lower price and a local market of recovered materials.

\section{Discussion and conclusions}

The consumption of EEE products is growing as fast as they are disposed. However, recovery of the WEEE is difficult due to multiple reasons. One of the most challenging factors is the lack of a collaborative approach to integrating different remanufacturing sectors. Targeting this very challenge, a novel cloud-based WEEE remanufacturing system, WRCloud, is developed. It offers cloudbased remanufacturing services, with data and knowledge wellmaintained throughout the whole product lifecycle.

In this research, the information control is supported by a QR code-based product tracking mechanism, which streamlines the WEEE data management. Foremost, it lowers the entry level of knowledge to the WEEE remanufacturing, and enables the general public to be involved in the extended WEEE recovery business. Combined with LCA mechanism, the system can offer optimised services based on quantitative assessment.

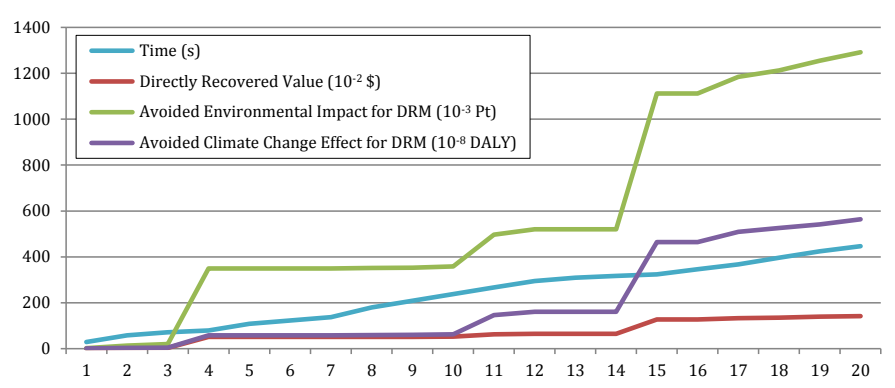

(a) Environmental and climate impact

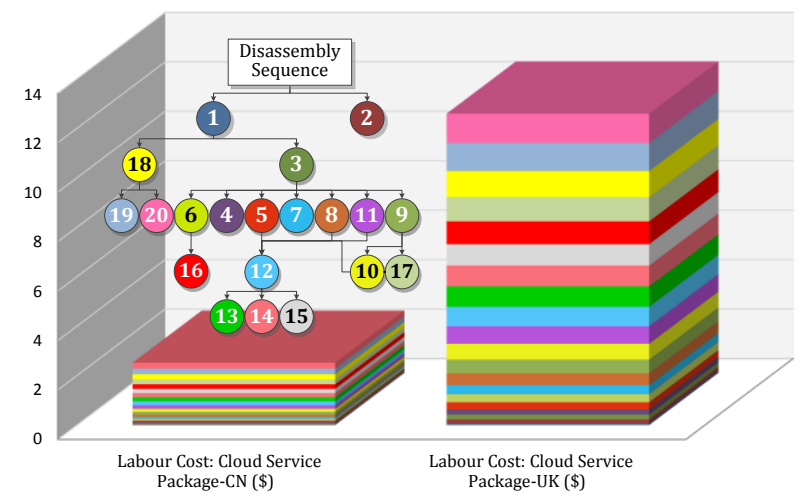

(b) Disassembly sequence and labour cost

Fig. 8. Service evaluation results of LCD TV disassembly.

Cloud remanufacturing is a promising approach to reshape the remanufacturing industry and to transform the current business model, thanks to its customised, distributed and scalable nature. However, the adoption of the cloud concept in the remanufacturing business could be challenging at the beginning, since there are extra configuration efforts needed for both manufacturers and recyclers before joining the cloud. Along with cloud computing's success, concerns have also been raised regarding the security and privacy leaks. For remanufacturing cloud, it is important to determine the right amount of data that is shared in the cloud, and maintain the access control for selective restriction of data and knowledge access.

In the future, it is possible to introduce more advanced cloud manufacturing technologies to remanufacturing, such as cloudbased disassembly, inventory monitoring and control by using auto-identifying techniques. All this will lead towards fully functioning cyber-physical production systems.

\section{References}

[1] US Environmental Protection Agency (2012) Statistics on the Management of Used and End-of-Life Electronics. Available from: http://www.epa.gov/wastes/ conserve/materials/recycling/manage.htm.

[2] Duflou JR, Seliger G, Kara S, Umeda Y, Ometto A, Willems B (2008) Efficiency and Feasibility of Product Disassembly: A Case-Based Study. CIRP Annals Manufacturing Technology. 57(2):583-600.

[3] Grunow M, Gobbi G (2009) Designing the Reverse Network for WEEE in Denmark. CIRP Annals - Manufacturing Technology. 58(1):391-394.

[4] Rahimifard S, Abu Bakar MS, Williams DJ (2009) Recycling Process Planning for the End-of-Life Management of Waste from Electrical and Electronic Equipment. CIRP Annals - Manufacturing Technology. 58(1):5-8.

[5] Nishino N, Wang S, Tsuji N, Kageyama K, Ueda K (2012) Categorization and Mechanism of Platform-Type Product-Service Systems in Manufacturing. CIRP Annals - Manufacturing Technology. 61(1):391-394.

[6] Mell P, Grance T (2011) The NIST Definition of Cloud Computing. NIST Special Publication. 800(145):7.

[7] Wang L (2013) Machine Availability Monitoring and Machining Process Planning towards Cloud Manufacturing. CIRP Journal of Manufacturing Science and Technology. 6(4):263-273.

[8] Wang XV, Xu X (2013) An Interoperable Solution for Cloud Manufacturing. Robotics and Computer-Integrated Manufacturing. 29(4):232-247.

[9] Perry N, Bernard A, Laroche F, Pompidou S (2012) Improving Design for Recycling - Application to Composites. CIRP Annals - Manufacturing Technology. 61(1):151-154.

[10] Váncza J, Monostori L, Lutters D, Kumara SR, Tseng M, Valckenaers P, Van Brussel H (2011) Cooperative and Responsive Manufacturing Enterprises. CIRP Annals - Manufacturing Technology. 60(2):797-820.

[11] Goedkoop M, Spriensma R (2001) The Eco-indicator99: A Damage Oriented Method for Life Cycle Impact Assessment. Methodology Annex. Available from: http://www.pre-sustainability.com/download/EI99_annexe_v3.pdf. 